\title{
Evolution of the velocity distribution of atoms under the action of the bichromatic force
}

\author{
V. I. Romanenkd* and L. P. Yatsenko \\ Institute of Physics of the National Academy of Sciences of Ukraine, \\ Nauky Avenue 46, Kyiv 03680, Ukraine
}




\begin{abstract}
We study numerically the evolution of the velocity distribution of atoms under the action of the bichromatic force. The comparison of the time dependencies of the distribution width and the average acceleration of atoms reveals the correlation of these quantities. We show that the estimation of the momentum diffusion coefficient on the basis of the analogy between the interaction of atoms with the counter-propagating bichromatic waves and the interaction of atoms with the counter-propagating sequences of the $\pi$-pulses roughly corresponds to the results of numerical calculations. To separate the influence of the momentum diffusion on the evolution of atomic momentum distribution from the influence of the time-dependent Doppler shift, we study the motion of a "heavy" atom, for which the velocity change during the interaction of an atom with the field can be neglected. Provided that the parameters of the atom-field interaction are optimal, we show that the momentum diffusion coefficient is proportional to the intensity of the laser radiation. We used the Monte Carlo wave-function method for the numerical simulation of the atomic motion.
\end{abstract}

\title{
CONTENTS
}

I. Introduction

II. Electric field and Hamiltonian

III. Schrödinger equation and modeling of the state vector by Monte Carlo wave function method

IV. Numerical calculation routine

V. Results of numerical simulations

A. Sodium and cesium atoms in the field of counter-propagating bichromatic waves 11

B. Heavy atoms in the field of counter-propagating bichromatic waves. Light pressure force and momentum variance

\footnotetext{
* Institute of Physics of the National Academy of Sciences of Ukraine, Nauky Avenue 46, Kyiv 03680, Ukraine; vr@iop.kiev.ua
} 


\section{INTRODUCTION}

The first theoretical study of the light pressure force exerted on atoms in the field of counter-propagating bichromatic waves in 1988 [1] showed that this force can be used to control the motion of atoms. This control is based on the large light pressure force exerted on atoms, which is much larger than the maximal light pressure force $F_{s p}$ in the field of a traveling monochromatic wave, given by the formula [2, 3]

$$
F=\frac{1}{2} \hbar k \gamma
$$

Here $\gamma$ is the rate of the spontaneous emission, $k$ is the wave vector. This fundamental limit was exceeded already in the first observation of the bichromatic force [4]. Later, the light pressure force much greater than $F_{s p}$ was experimentally confirmed [5]. Analytical theory of the bichromatic force was developed in the works [6, 7], a review of publications on this topic are given in [8]. At the same time, momentum diffusion of atoms in the field of counter-propagating bichromatic waves has not been studied so far, in contrast to wellknown thorough studies of momentum diffusion of atoms in the field of monochromatic waves [2, 9] and momentum diffusion of atoms in the field of $\pi$-pulses [10].

In this paper, we obtain statistical characteristics of the atomic ensemble in the field of bichromatic force by numerical simulation of the atomic state vector of each atom using the Monte Carlo wave function method [11, 12], followed by averaging over all the atoms in the ensemble. We show that only at the beginning of the interaction of the atoms with the field the time evolution of the width $\Delta p$ of the momentum distribution of the atoms can be described by the diffusion-like dependence $\Delta p=\sqrt{2 D t}$, where $D$ is the momentum diffusion coefficient and $t$ is the atom-field interaction time. Over time, the width $\Delta p$ can both increase and decrease depending on the change of the average velocity of atoms.

To separate the influence of momentum diffusion on the evolution of momentum distribution of atoms from the influence of the Doppler shift of the atomic frequency, we used an assumption of a "heavy" atom, when the change of velocity during the interaction of atom with the field can be neglected. This allowed us to study the dependencies of the momentum 
diffusion coefficient on the average velocity of atoms and the momentum diffusion coefficient on the intensity of laser radiation.

The paper is structured as follows. The next section presents the equations that describe the time dependence of the field acting on the atoms and the Hamiltonian of the atom-field interaction. The third section describes the Monte Carlo method for the wave function. In the fourth section, we present the scheme of the numerical calculation. The obtained results are discussed in the fifth section. In the sixth section, we formulate brief conclusions of the work.

\section{ELECTRIC FIELD AND HAMILTONIAN}

Let's consider a two-level atom with the ground $|g\rangle$ and the excited $|e\rangle$ states, which interacts with two counter-propagating bichromatic waves

$$
\boldsymbol{E}_{p}=\frac{1}{2} \boldsymbol{e} E_{0} \cos \left[\left(\omega+\frac{1}{2} \Omega\right) t+\frac{\varphi}{2}-k z\right]+\frac{1}{2} \boldsymbol{e} E_{0} \cos \left[\left(\omega-\frac{1}{2} \Omega\right) t-\frac{\varphi}{2}-k z\right]
$$

and

$$
\boldsymbol{E}_{m}=\frac{1}{2} \boldsymbol{e} E_{0} \cos \left[\left(\omega+\frac{1}{2} \Omega\right) t-\frac{\varphi}{2}+k z\right]+\frac{1}{2} \boldsymbol{e} E_{0} \cos \left[\left(\omega-\frac{1}{2} \Omega\right) t+\frac{\varphi}{2}+k z\right],
$$

where $E_{0}$ is the peak to peak amplitude of the waves which is assumed to be the same for

all waves, $\boldsymbol{e}$ is the unit polarization vector, $\omega \pm \frac{1}{2} \Omega$ are the carrier frequencies with the mean frequency $\omega$ and the difference $\Omega$.

Here we neglected the difference of the wave vectors $\sim(\Omega / \omega) k$; taking it into account leads to a change in the phase difference which is substantial at a distance of the order $(\omega / \Omega) \lambda \sim\left(10^{6} \div 10^{7}\right) \lambda \sim 1 \mathrm{~m}$, where $\lambda$ is the wavelength of electromagnetic radiation. In our model, used in other works $[1,4 \underline{4}, \underline{6}, 10,13,14]$, we describe the phase difference between the waves by the terms $\pm \varphi / 2$.

The Hamiltonian of an atom in the field

$$
E=E_{p}+E_{m}
$$

has the form

$$
H=\frac{\hat{\boldsymbol{p}}^{2}}{2 M}+\hbar \omega_{0}|e\rangle\langle e|-\hat{\boldsymbol{d}} \cdot \boldsymbol{E} .
$$

Here $\hat{\boldsymbol{p}}$ is the momentum operator of the atom, $M$ is the atomic mass, $\omega_{0}$ is the transition frequency (energy difference of the states $e$ and $g$ ), $\hat{\boldsymbol{d}}$ is the dipole momentum operator. 
The field (4) can be written as counter-propagating amplitude-modulated waves

$$
\boldsymbol{E}=\boldsymbol{e} E_{1} \cos (\omega t-k z)+\boldsymbol{e} E_{2} \cos (\omega t+k z),
$$

where

$$
\begin{aligned}
& E_{1}=E_{0} \cos \left(\frac{1}{2} \Omega t+\frac{1}{2} \varphi\right) ; \\
& E_{2}=E_{0} \cos \left(\frac{1}{2} \Omega t-\frac{1}{2} \varphi\right) .
\end{aligned}
$$

Here $\frac{1}{2} \Omega$ is the modulation frequency (half the difference of the frequencies of monochromatic waves $\Omega$ that form bichromatic waves (21), (3)). The field (4) can also be interpreted as a bichromatic standing wave

$$
E=E_{0} \cos \left(k z+\frac{\varphi}{2}\right) \cos \left[\left(\omega-\frac{1}{2} \Omega\right) t\right]+E_{0} \cos \left(k z-\frac{\varphi}{2}\right) \cos \left[\left(\omega+\frac{1}{2} \Omega\right) t\right] .
$$

\section{SCHRÖDINGER EQUATION AND MODELING OF THE STATE VECTOR BY MONTE CARLO WAVE FUNCTION METHOD}

We determine the temporal evolution of the state vector $|\psi\rangle$ from the Schödinger equation

$$
i \hbar \frac{d}{d t}|\psi\rangle=H|\psi\rangle-i \hbar \frac{\gamma}{2}|e\rangle\langle e \mid \psi\rangle
$$

by the Monte Carlo wave function method [11, 12].

The last term in Eq. (101) describes the spontaneous emission by the atom in the excited state with the rate $\gamma$. To reduce the number of equations needed to describe the evolution of the state vector, we assume, as in [12], that the momentum of the atom along the $O z$ axis is changed after spontaneous emission by $\pm \hbar k$ or does not change at all (the photon is emitted in the orthogonal to $O z$ axis direction).

We seek for the state vector in the form

$$
|\psi\rangle=c_{g}(z, t)|g\rangle+c_{e}(z, t) e^{-i \omega_{0} t}|e\rangle .
$$

Substituting (6) and (11) in (10), we find the equations for $c_{g}(z, t), c_{e}(z, t)$ in the rotating wave approximation [15]:

$$
\begin{aligned}
& i \frac{\partial}{\partial t} c_{g}=-\frac{\hbar}{2 M} \frac{\partial^{2}}{\partial z^{2}} c_{g}+\frac{1}{2}\left(V_{1} e^{i \eta_{1}}+V_{2} e^{i \eta_{2}}\right) c_{e}, \\
& i \frac{\partial}{\partial t} c_{e}=-\frac{\hbar}{2 M} \frac{\partial^{2}}{\partial z^{2}} c_{e}+\frac{1}{2}\left(V_{1} e^{-i \eta_{1}}+V_{2} e^{-i \eta_{2}}\right) c_{g}-i \frac{\gamma}{2} c_{e} .
\end{aligned}
$$


Here $\eta_{1}=\left(\omega-\omega_{0}\right) t-k z, \eta_{2}=\left(\omega-\omega_{0}\right) t+k z$ and $V_{1}, V_{2}$ are defined by expressions

$$
\begin{aligned}
& V_{1}=-\frac{1}{\hbar}\langle g|\hat{\boldsymbol{d}} \cdot \boldsymbol{e}| e\rangle E_{1}, \\
& V_{2}=-\frac{1}{\hbar}\langle g|\hat{\boldsymbol{d}} \cdot \boldsymbol{e}| e\rangle E_{2} .
\end{aligned}
$$

To simplify the notations, in (12), (13) and hereafter we omit the arguments denoting the dependence of quantities on time and coordinates.

We write $c_{g}, c_{e}$ in the form

$$
\begin{aligned}
c_{g} & =\sum_{-\infty}^{\infty} b_{g, n} \exp \left(\frac{i}{2} \delta t+i k_{0} z-i \frac{\hbar k_{0}^{2}}{2 M} t\right)\langle z \mid n\rangle, \\
c_{e} & =\sum_{-\infty}^{\infty} b_{e, n} \exp \left(-\frac{i}{2} \delta t+i k_{0} z-i \frac{\hbar k_{0}^{2}}{2 M} t\right)\langle z \mid n\rangle,
\end{aligned}
$$

where $\delta=\omega-\omega_{0}$ is the detuning of the average frequence of the bichromatic wave from the transition frequency in the atom, $\langle z \mid n\rangle=e^{i n k z}, k_{0}=p_{0} / \hbar, p_{0}$ is $z$ component of the initial momentum of the atom along the axis $O z$. Time-dependent phases in Eqs. (16), (17) do not influence the probabilities $b_{g, n}, b_{e, n}$ to find the atom in the states $|g, n\rangle=|g\rangle \otimes|n\rangle$, $|e, n\rangle=|e\rangle \otimes|n\rangle$.

To obtain the equations for $b_{g, n}, b_{e, n}$, we substitute equation (16), (17) in equations (12), (13):

$$
\begin{aligned}
& \frac{\partial}{\partial t} b_{g, n}=-i\left(n^{2} \delta_{r e c}+\frac{n \hbar k k_{0}}{M}+\frac{1}{2} \delta\right) b_{g, n}-\frac{i}{2}\left(V_{1} b_{e, n+1}+V_{2} b_{e, n-1}\right) \\
& \frac{\partial}{\partial t} b_{e, n}=-i\left(n^{2} \delta_{r e c}+\frac{n \hbar k k_{0}}{M}-\frac{1}{2} \delta\right) b_{e, n}-\frac{i}{2}\left(V_{1} b_{g, n-1}+V_{2} b_{g, n+1}\right)-\frac{\gamma}{2} b_{e, n} .
\end{aligned}
$$

Here $\delta_{\text {rec }}=\hbar k^{2} /(2 M)$.

Hereinafter we assume that $\left\langle g\left|\hat{\boldsymbol{d}} \cdot \boldsymbol{e}_{1}\right| e\right\rangle=\left\langle g\left|\hat{\boldsymbol{d}} \cdot \boldsymbol{e}_{2}\right| e\right\rangle$, then

$$
\begin{aligned}
& V_{1}=\Omega_{0} \cos \left(\frac{1}{2} \Omega t+\frac{1}{2} \varphi\right) \\
& V_{2}=\Omega_{0} \cos \left(\frac{1}{2} \Omega t-\frac{1}{2} \varphi\right),
\end{aligned}
$$

where $\Omega_{0}=-\langle g|\hat{\boldsymbol{d}} \cdot \boldsymbol{e}| e\rangle E_{0} / \hbar$.

We seek for the state vector (11) by the Monte Carlo wave function method [11, 12]. This method, when applied to the amplitudes of the probabilities $b_{g, n}, b_{e, n}$ to find an atom in the ground or excited states with momentum $\hbar k_{0}+n \hbar k$, looks like the following. 
1. We assume that at time $t$ the amplitudes $b_{g, n}(t), b_{e, n}(t)$ are normalized:

$$
\sum_{n=-\infty}^{\infty}\left(\left|b_{g, n}(t)\right|^{2}+\left|b_{e, n}(t)\right|^{2}\right)=1
$$

Knowing $b_{g, n}(t), b_{e, n}(t)$, we find the values of $b_{g, n}^{(1)}(t+\Delta t), b_{e, n}^{(1)}(t+\Delta t)$ from Eqs. (18), (19) for a small time interval $\Delta t$. The presence of a dissipative term in (10) leads to a violation of the state vector normalization, therefore with the change of time $t \rightarrow t+\Delta t$ the equality (22) is violated. For a difference

$$
1-\sum_{n=-\infty}^{\infty}\left(\left|b_{g, n}^{(1)}(t+\Delta t)\right|^{2}+\left|b_{e, n}^{(1)}(t+\Delta t)\right|^{2}\right)=\Delta P
$$

we have

$$
\Delta P=\gamma \Delta t \sum_{n=-\infty}^{\infty}\left|b_{e, n}^{(1)}(t)\right|^{2}
$$

The meaning of this equality is obvious: the factor $\sum_{n=-\infty}^{\infty}\left|b_{e, n}^{(1)}(t)\right|^{2}$ is the population of the excited state of the atom. Therefore, $\Delta P$ is the probability of an atom to emit a photon during the time interval $\Delta t$.

2. To find whether there was a photon emission during the time $\Delta t$, we generate a random variable $\epsilon$ which is uniformly distributed between zero and one and compare it with $\Delta P$. If $\epsilon>\Delta P$ (in most cases), no emission of photon has occurred. Then we form the probability amplitudes $b_{g, n}(t+\Delta t), b_{e, n}(t+\Delta t)$ at time $t+\Delta t$ by normalizing the values $b_{g, n}^{(1)}(t+\Delta t), b_{e, n}^{(1)}(t+\Delta t)$ that where found in the first stage:

$$
\begin{aligned}
& b_{g, n}(t+\Delta t)=\frac{b_{g, n}^{(1)}(t+\Delta t)}{\sqrt{1-\Delta P}}, \\
& b_{e, n}(t+\Delta t)=\frac{b_{e, n}^{(1)}(t+\Delta t)}{\sqrt{1-\Delta P}} .
\end{aligned}
$$

If $\epsilon \leq \Delta P$, the atom emits a photon and goes to the ground state. In this case, after the emission of a photon $b_{g, n}(t+\Delta t)$ and $b_{e, n}(t+\Delta t)$ are defined as follows:

$$
\begin{aligned}
& b_{g, n}(t+\Delta t)=\frac{b_{e, n-\xi}^{(1)}(t+\Delta t)}{\sum_{m=-\infty}^{\infty}\left|b_{e, m}^{(1)}(t+\Delta t)\right|^{2}} \\
& b_{e, n}(t+\Delta t)=0,
\end{aligned}
$$


where $\xi$ takes one of the values $\xi=0, \pm 1$ with some probability. Recall, that we simulate the real distribution of the projections of the photon momentum on the $\mathrm{Oz}$ axis by a hypothetical distribution when $\xi=0, \pm 1$ (as if atoms emit photons either in the direction of the $O z$ axis or perpendicular to it), as was done in the modeling of Doppler cooling in [12, 16]. In this case, we have a discrete distribution of atoms according to the projection of the spontaneous photon momentum on the axis $O z$ with the step $\hbar k$.

Instead of going back to step 1 and continuing the calculation further, we adjust the numbering of the amplitudes $b_{g, n}(t+\Delta t)$ in order to reduce the required size of the amplitude arrays in numerical calculations. A monotonic change of the average momentum of an atom over time (in the classical description of the motion of an atom, a force acts on it) increases the size of arrays of the probability amplitudes required for calculations. To reduce the size of the arrays, we calculate the average momentum acquired by the atom between the moments of spontaneous photon emission

$$
\langle\Delta p\rangle=\hbar k \sum_{n=-\infty}^{\infty} n\left|b_{g, n}\right|^{2},
$$

and find the integer number $N$ of photon momentum $\hbar k$ which it contains

$$
N=\left[\frac{\langle\Delta p\rangle}{\hbar k}\right],
$$

where square brackets denote an integer part of a number. Next, we change the numbering of the amplitudes

$$
b_{g, n} \rightarrow b_{g, n-N} .
$$

This is equivalent to changing the momentum of an atom to $-N \hbar k$, so we also change the momentum

$$
p_{0} \rightarrow p_{0}+N \hbar k,
$$

so that the distribution of atoms by pulses does not change, and return to step 1.

For definiteness, we assume that the atom in the ground and excited states is characterized by complete momentums $\hbar J_{g}, \hbar J_{e}$ with $J_{e}=J_{g}+1$. In this case, the two-level scheme of the atom-light interaction between the states $\left|g, m_{g}=J_{g}\right\rangle$ and $\left|e, m_{e}=J_{e}\right\rangle$ (these are the states that we denoted for simplicity by $|g\rangle,|e\rangle)$ is realized when the atom interacts with circularly 
polarized light. The optimal description of the momentum diffusion rate due to spontaneous emission by a discrete distribution of the projection of the photon momentum on the axis $O z$ occurs if $\xi$ acquires the values $-1,0,+1$ with probabilities of $1 / 5,3 / 5,1 / 5$ [12, 16]. In essence, this means that the specified distribution law for $\xi$ gives the same mean value of the square of the projection of the photon momentum on the $O z$ axis as the real distribution of the projection of the photon momentum on the $O z$ axis.

In the calculations, we will also use the model of the heavy atom, $M \rightarrow \infty$. In this case, the equations (18), (19) read

$$
\begin{aligned}
\frac{\partial}{\partial t} b_{g, n} & =\left(-i n k v-\frac{i}{2} \delta\right) b_{g, n}-\frac{i}{2}\left(V_{1} b_{e, n+1}+V_{2} b_{e, n-1}\right) \\
\frac{\partial}{\partial t} b_{e, n} & =\left(-i n k v+\frac{i}{2} \delta\right) b_{e, n}-\frac{i}{2}\left(V_{1} b_{g, n-1}+V_{2} b_{g, n+1}\right)-\frac{\gamma}{2} b_{e, n}
\end{aligned}
$$

where $v$ is the velocity of the atom which in the approximation $M \rightarrow \infty$ does not depend on time.

\section{NUMERICAL CALCULATION ROUTINE}

We consider an ensemble of $N$ atoms in the field of the bichromatic counter-propagating waves and assume that each atom begins to move with a projection $v_{0}$ of the initial velocity on the axis $O z$. The evolution of the state vector of the atom is calculated by the procedure described in section [II]. We repeat it many times until we reach the final time of calculation $t_{f}$.

Knowing the final state vector of each of the $N$ atoms, we determine the average momentum of the $m$-th atom by the formula

$$
\left\langle p^{(m)}\right\rangle=p_{0}^{(m)}+\hbar k \sum_{n=-\infty}^{\infty} n\left(\left|b_{g, n}^{(m)}\right|^{2}+\left|b_{e, n}^{(m)}\right|^{2}\right),
$$

where the values of the probability amplitudes refer to the $m$-th atom, and $p_{0}^{(m)}$ is the initial value of the momentum after the last act of spontaneous radiation, which is modified after

each spontaneous photon radiation according to (33). The average value of the square of the momentum of the $m$-th atom is calculated by the formula

$$
\left\langle\left(p^{(m)}\right)^{2}\right\rangle=\sum_{n=-\infty}^{\infty}\left(p_{0}^{(m)}+n \hbar k\right)^{2}\left(\left|b_{g, n}^{(m)}\right|^{2}+\left|b_{e, n}^{(m)}\right|^{2}\right) .
$$


Now we can calculate the average value of $z$-component of the momentum per an atom in the ensemble

$$
p_{a v}=\frac{1}{N} \sum_{m=-\infty}^{\infty}\left\langle p^{(m)}\right\rangle
$$

and the standard deviation of the $z$-component of the momentum

$$
\Delta p=\sqrt{\frac{\sum_{m=-\infty}^{\infty}\left\langle\left(p^{(m)}\right)^{2}\right\rangle}{N}-p_{a v}^{2}}
$$

per an atom from its average value. Eqs. (38), (39) allow us to calculate the average force $F$ acting on the atom and the momentum diffusion coefficient $D$ :

$$
\begin{aligned}
& F=\frac{p_{a v}}{t_{f}}, \\
& D=\frac{\Delta p^{2}}{2 t_{f}}
\end{aligned}
$$

In the following calculations, we will compare the force of light pressure on the atom in the field of counter-propagating bichromatic waves with the maximal force $F_{s p}$ of pressure on the atom in the field of the monochromatic traveling wave given by Eq. (1). The momentum diffusion coefficient we will compare with the maximal momentum diffusion coefficient $D_{r}$ in the field of a traveling monochromatic wave along the direction of its propagation [2]:

$$
D_{r}=\frac{1}{4} \hbar^{2} k^{2} \gamma(1+\alpha)
$$

where $\alpha=\left\langle\cos ^{2} \theta\right\rangle$ is the mean value of the square of the cosine of the angle between the direction of photon radiation and the direction of wave propagation. For the model that we adopted here $\alpha=2 / 5$.

\section{RESULTS OF NUMERICAL SIMULATIONS}

We perform numerical simulation of temporal evolution of the average velocity and the variance of the velocity of atoms in the field of counter-propagating bichromatic waves for sodium and cesium atoms. In addition, we consider the force of light pressure acting on atoms and the variance of the velocity of atoms for the limit case of very heavy atoms, when the change of the velocity of an atom (but not the momentum of the atom) during its interaction with the field can be neglected. 
Calculations were carried out for atoms ${ }^{23} \mathrm{Na}$ and ${ }^{133} \mathrm{Cs}$, in which a cyclic interaction with the field can be created [3]. The wavelength of the transition $3^{2} S_{1 / 2}-3^{2} P_{3 / 2}$ in the sodium atom is $\lambda=589.16 \mathrm{~nm}$, the rate of spontaneous emission is $\gamma=2 \pi \times 9.795 \mathrm{MHz}$, the Doppler cooling limit is $T_{D}=235.03 \mu \mathrm{K}$ [17]. In a cesium atom, the wavelength of the transition $6^{2} S_{1 / 2}-6^{2} P_{3 / 2}$ is $\lambda=852.35 \mathrm{~nm}$, the rate of spontaneous emission is $\gamma=2 \pi \times 5.23 \mathrm{MHz}$, the Doppler limit of atomic cooling is $T_{D}=125.61 \mu \mathrm{K}$ [18]. All calculations were performed for the case $\omega=\omega_{0}$, i.e. $\delta=0$.

\section{A. Sodium and cesium atoms in the field of counter-propagating bichromatic} waves

Fig. 1 shows time evolution of the mean velocity $\bar{v}$ and the standard deviation of the velocity $\Delta v$ from its mean value (square root of the velocity variance) for sodium atoms in the field of counter-propagating bichromatic waves. The plots are obtained both for the optimal ratio of the Rabi frequency $\Omega_{0}$ to the difference of the frequencies of monochromatic waves $\Omega$ that form bichromatic waves at $\varphi=\pi / 4\left(\Omega_{0}=\sqrt{3 / 2} \Omega[6]\right)$ and for a small but noticeable $(\approx 20 \%)$ deviation from the optimum. First of all, it should be noted that with increasing time the velocity of the atom approaches $v=\Omega /(2 k)$, when, according to the quasi-classical theory [1, 5], the force of light pressure exerted on the atom is zero. The standard deviation $\Delta v$ of velocity from the mean value of $\bar{v}$ increases monotonically with time only at the beginning of the interaction of atoms with the field, then this dependence becomes nonmonotonic. This suggests that influence of the Doppler effect, which becomes more noticeable with increasing velocity, on the distribution of atoms in the momentum space is significant. Also, the Doppler effect leads to a change in the almost linear dependence of velocity on time at the beginning of the interaction with the field (which indicates an almost constant value of the light pressure force exerted on the atom) to nonlinear. In the next section, to exclude the influence of the time-dependent Doppler shift on the light pressure force and the momentum dispersion, we consider the interaction of atoms of very large mass with counter-propagating bichromatic waves.

Fig. 2, $a$ shows the time dependence of the acceleration $a=d \bar{v} / d t$, calculated by differentiation of the time dependence of the average velocity, which is depicted in Fig. 1, $a$, after its pre-smoothing with the program GNUPLOT (option acsplines with smoothing parameter 

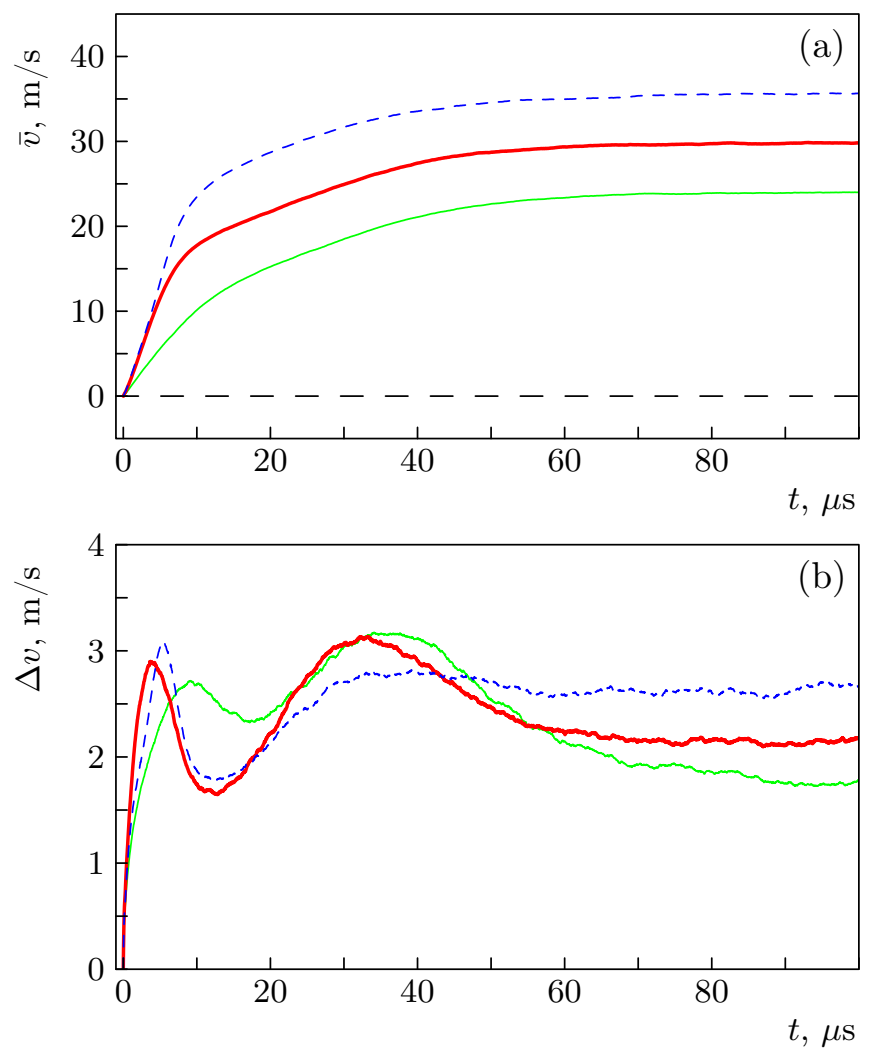

FIG. 1. Time dependence of the average velocity $\bar{v}(\mathrm{a})$ and the square root of the velocity variance $\Delta v$ (b) for 1000 sodium atoms in the field of the counter-propagatiing bichromatic waves. Parameters are: $\Omega_{0}=2 \pi \times 122 \mathrm{MHz} ; \Omega=2 \pi \times 80 \mathrm{MHz}$ (thin curve), $\Omega=2 \pi \times 100 \mathrm{MHz}$ (thick curve), $\Omega=2 \pi \times 120 \mathrm{MHz}$ (dashed curve). The initial velocity of the atoms is $v_{0}=0$. The phase difference of the counter-propagating waves is $\varphi=\pi / 4$

1). Such smoothing is necessary because the derivative of the unsmoothed dependence of $\bar{v}$ on time has peculiarities due to quantum jumps in the atoms. A mechanical analog of this averaging procedure is the movement of the piston in a cylinder filled with some gas under the action of molecules. Note that the smoothed dependence of $\bar{v}$ on time is visually indistinguishable from the non-smoothed dependence of $\bar{v}$ which is shown in Fig. 1, $a$. The maximum average acceleration of atoms in Fig. 2 is more than three times higher than the maximum acceleration of the atom in the field of a traveling light wave, which for the sodium atom is $a_{s p}=F_{s p} / m=\hbar k \gamma /(2 m)=0.925 \cdot 10^{6} \mathrm{~m} / \mathrm{s}^{2}$, which roughly corresponds to the results of [1] obtained for the model of the "heavy" atom.

Comparing the time dependence of the average velocity $\bar{v}$ of atoms against the smoothed time dependence of the acceleration, one can obtain the dependence of the average acceler- 

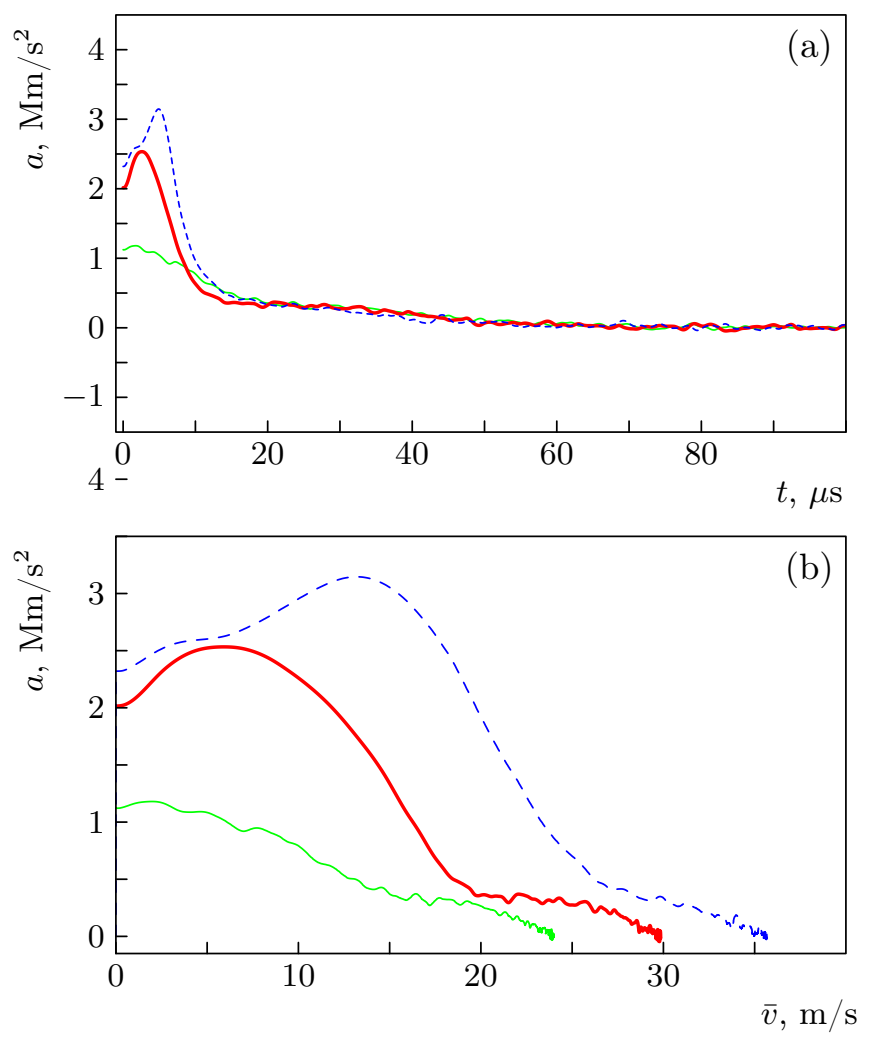

FIG. 2. Time dependence of the average acceleration $a=d \bar{v} / d t$ (a) and the dependence of the average acceleration $a$ on the average velocity $\bar{v}$ of atom (b) for 1000 sodium atoms in the field of counter-propagating bichromatic waves. Parameters are: $\Omega_{0}=2 \pi \times 122 \mathrm{MHz} ; \Omega=2 \pi \times 80 \mathrm{MHz}$ (thin curve), $\Omega=2 \pi \times 100 \mathrm{MHz}$ (thick curve), $\Omega=2 \pi \times 120 \mathrm{MHz}$ (dashed curve). The phase difference of the counter-propagating waves is $\varphi=\pi / 4$

ation $a$ of atoms on the average velocity (see Fig. 2, $b$ ). The fluctuation-like dependence of acceleration at high atomic velocities, when the value of $k \bar{v}$ is close to $\Omega / 2$, is due to the relatively small number of atoms in our calculations.

In Fig. 3 we show the initial part of the dependence of $\Delta v$ on $\bar{v}$ in Fig. 1. At the beginning of the interaction of atoms with the field, the calculated dependencies of $\Delta v$ on $t$ are well approximated by the curves $\Delta v=\sqrt{2 D_{v} t}$. This indicates the diffusion nature of the spreading of the distribution of atoms by momentum at the beginning of their interaction with the field. For a larger time, the dependence of $\Delta v$ on time is no longer described by the diffusion law that can be explained by the dependence of the diffusion coefficient $D_{v}$ on the velocity of the atom.

The diffusion coefficient $D_{v}$ in the velocity space is related to the momentum diffusion 
coefficient $D$ by the relation:

$$
D_{v}=D / m^{2}
$$

Let us compare the diffusion coefficient in the velocity space in the field of counterpropagating bichromatic waves with that in the field of a traveling monochromatic wave of high intensity (when there is a saturation of absorption)

$$
D_{v r}=D_{r} / m^{2}
$$

where $D_{r}$ is given by the expression (42). Calculations for sodium atoms with $\alpha=0.4$ give $D_{r}=2.78 \cdot 10^{-47} \mathrm{~kg}^{2} \mathrm{~m}^{2} / \mathrm{s}^{3}, D_{v r}=19.07 \cdot 10^{3} \mathrm{~m}^{2} / \mathrm{s}^{3}$. For the curves shown in Fig. 3 we have the ratio $D_{v} / D_{v r}: 34.5(\Omega / 2 \pi=80 \mathrm{MHz}), 60.6(\Omega / 2 \pi=100 \mathrm{MHz}), 47.9(\Omega / 2 \pi=120 \mathrm{MHz})$, i.e. the coefficient of momentum diffusion of sodium atoms in the field of counter-propagating bichromatic waves exceeds the coefficient of momentum diffusion in the field of traveling monochromatic wave by $1-2$ orders.

Now we compare the calculated momentum diffusion coefficients with a rough estimate of the diffusion coefficient, which can be obtained by a close analogy between the interaction of atoms with sequences of counter-propagating $\pi$-pulses and the bichromatic field [1, 4 6,10$]$.

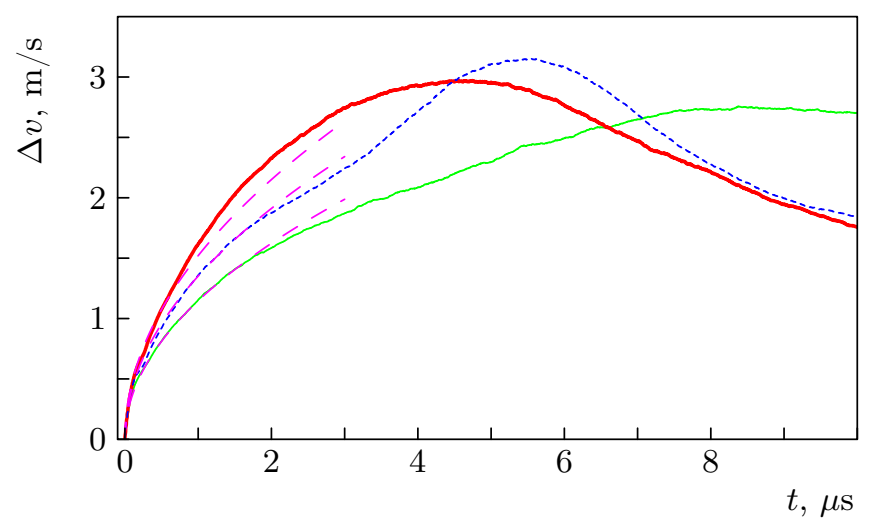

FIG. 3. Time dependence of the standard deviation $\Delta v$ of the velocity of atoms on its average value $\bar{v}$ for 1000 sodium atoms in the field of counter-propagating bichromatic waves. Parameters are: $\Omega_{0}=2 \pi \times 122 \mathrm{MHz} ; \Omega=2 \pi \times 80 \mathrm{MHz}$ (thin curve), $\Omega / 2 \pi=100 \mathrm{MHz}$ (thick curve), $\Omega=2 \pi \times 120 \mathrm{MHz}$ (short dashed curve). The initial velocity of the atoms is $v_{0}=0$. The long dashed curve shows the dependencies $\Delta v=\sqrt{2 D_{v} t}$, close to the curves with $\Omega=2 \pi \times 80 \mathrm{MHz}\left(D_{v}=0.6584 \cdot 10^{6} \mathrm{~m}^{2} / \mathrm{s}^{3}\right)$, $\Omega=2 \pi \times 100 \mathrm{MHz}\left(D_{v}=1.1562 \cdot 10^{6} \mathrm{~m}^{2} / \mathrm{s}^{3}\right), \Omega=2 \pi \times 120 \mathrm{MHz}\left(D_{v}=0.9135 \cdot 10^{6} \mathrm{~m}^{2} / \mathrm{s}^{3}\right)$. Phase difference of the counter-propagating waves is $\varphi=\pi / 4$ 
In paper [10], we found the coefficient of diffusion of atoms in the field of counter-propagating sequences of $\pi$-pulses with the repetition period $T$ for the model of a "heavy" atom, the maximum value of which (for $\tau=\frac{1}{2} T, \tau$ is the time shift between opposing pulses at the location of the atom) in the most interesting case $\gamma T \ll 1$ reaches

$$
D_{\pi \max }=\frac{4 \hbar^{2} k^{2}}{\gamma T^{2}} .
$$

Maximal value of the light pressure force in the field of the counter-propagating bichromatic waves

$$
F_{b i}=\frac{\hbar k}{\pi} \Omega
$$

may achieve the maximal light pressure force in the field of the sequences of the counterpropagating $\pi$-pulses with a repetition period $T=2 \pi / \Omega[\underline{5}, \underline{6}]$. This formula is valid provided that the optimal parameters of interaction of atoms with the field are chosen [6], in particular, for $\Omega_{0}=\sqrt{\frac{3}{2}} \Omega$ and $\varphi=\frac{1}{4} \pi$, if the atom occupies only one of the "dressed" states. In the general case, the real light pressure force exerted on atoms, taking into account the distribution of atoms by states, can be calculated only numerically [6].

For a rough estimation of the momentum diffusion coefficient in the field of the counterpropagating bichromatic waves, we use the analogy between the interaction of atoms with sequences of counter-propagating $\pi$-pulses and the interaction of atoms with the counterpropagating bichromatic waves. Substituting $T=2 \pi / \Omega$ in (45), we obtain a rough estimation of the momentum diffusion coefficient of atoms in the field of the counter-propagating bichromatic waves:

$$
D_{b i}=\frac{\hbar^{2} k^{2} \Omega^{2}}{\gamma \pi^{2}} .
$$

For sodium atoms Eq. (47) gives $D_{b i}=8.04 \cdot 10^{-46} \mathrm{~kg}^{2} \mathrm{~m}^{2} / \mathrm{s}^{3}$ for $\Omega=2 \pi \times 100 \mathrm{MHz}$. Estimation of the momentum diffusion coefficient from Fig. 3 for $\Omega=2 \pi \times 100 \mathrm{MHz}$ gives $D=1.7 \cdot 10^{-45} \mathrm{~kg}^{2} \mathrm{~m}^{2} / \mathrm{s}^{3}$, that is twice $D_{b i}$. The relatively small difference between $D$ and $D_{b i}$ indicates the expediency of using formula (47) for rough estimation of the momentum diffusion coefficient in the field of counter-propagating bichromatic waves provided that the optimal parameters of the atom-field interaction are chosen.

To estimate the influence of the initial velocity $v_{0}$ on the mean acceleration $a$ of the atom's motion and the standard deviation of the velocity from its mean value, it is convenient for each of the pairs of dependencies $a(t), \bar{v}(t)$ and $\Delta v(t), \bar{v}(t)$, calculated for the motion of 
the ensemble of atoms in the field of counter-propagating bichromatic waves, to construct curves whose abscissas are equal to $\bar{v}$, and the ordinates are $a$ (Fig. 4, $a$ ), and $\Delta v$ (Fig. 4, $b)$. To simplify the terminology, we will talk about these curves as dependences of $a$ and $\Delta v$ on $\bar{v}$. The physical basis of these dependencies is different conditions for the interaction
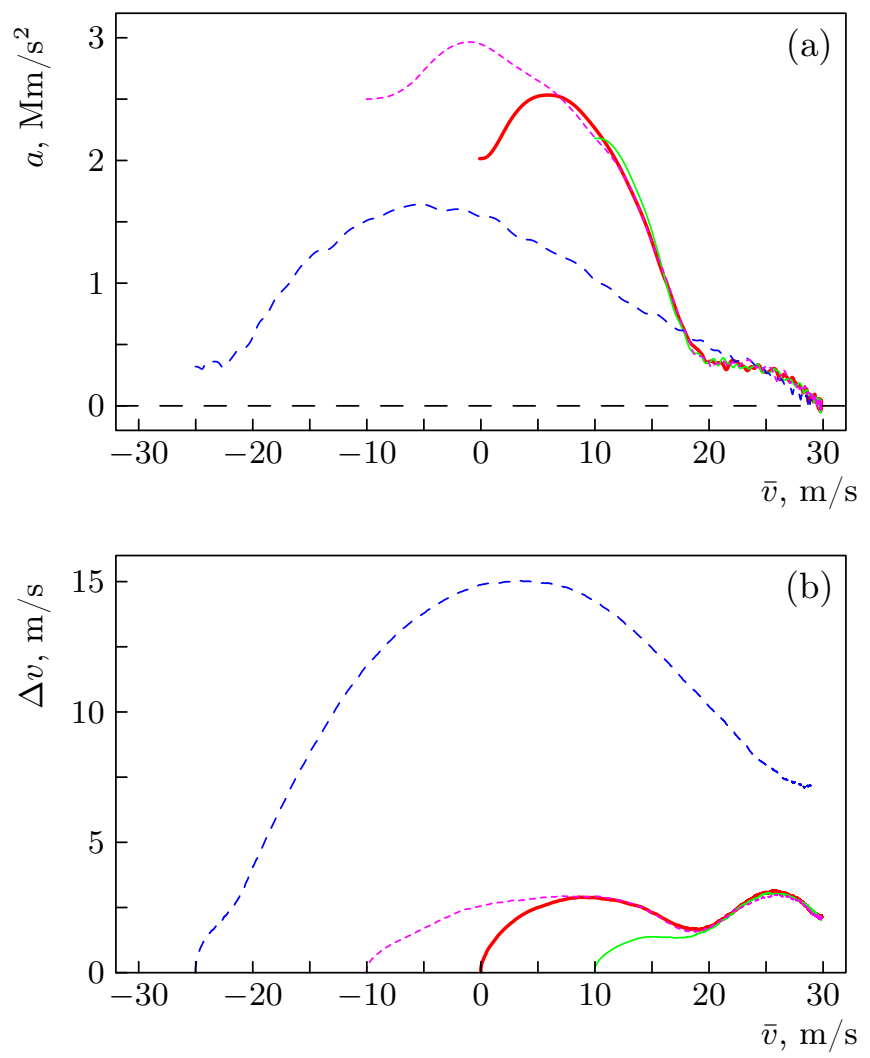

FIG. 4. Dependence of the average acceleration $a=d \bar{v} / d t$ on the average velocity $\bar{v}$ of atoms, calculated after smoothing $\bar{v}$ by the program GNUPLOT (a) and the dependence the standard deviation of the velocity $\Delta v$ from its mean value $\bar{v}$ on the average velocity $\bar{v}$ of atoms (b) for 1000 sodium atoms in the field of counter-propagating bichromatic waves for different values $v_{0}$ of the initial velocity of atoms. Parameters are: $\Omega_{0}=2 \pi \times 122 \mathrm{MHz} ; \Omega=2 \pi \times 100 \mathrm{MHz}$; thick solid curve corresponds to $v_{0}=0$, thin solid curve corresponds to $v_{0}=10 \mathrm{~m} / \mathrm{s}$, short dashed curve corresponds to $v_{0}=-10 \mathrm{~m} / \mathrm{s}$, long dashed curve corresponds to $v_{0}=-25 \mathrm{~m} / \mathrm{s}$. Phase difference of counter-propagating waves is $\varphi=\pi / 4$

of atoms with the field due to the Doppler effect and different initial conditions. Calculation of the average acceleration of atoms is performed by differentiating the dependence of the average velocity of atoms on time after its pre-smoothing using the program GNUPLOT 
(option acsplines with smoothing parameter 1). We also calculated the momentum diffusion coefficient for $v_{0}=-25 \mathrm{~m} / \mathrm{s}$, using the dependence $\Delta v(t)$ not given here and obtained $D_{v}=0.34 \cdot 10^{6} \mathrm{~m}^{2} / \mathrm{s}^{3}$, which is half as much as that at $v_{0}=0$, given in the caption to Fig. 3.

Shown in Fig. 4, $a$ dependencies of $a$ on $\bar{v}$ for $v_{0}=0, \pm 10 \mathrm{~m} / \mathrm{s}$ in common areas of definition differ a little, that is natural to expect, paying attention to the dependencies $\Delta v(\bar{v})$ in Fig. 4, $b$. The variation $k \Delta v$ of the Doppler shifts of the frequency of atoms calculated using these dependencies is much smaller than the average value of the Doppler shift $k \bar{v}$, so the atoms in the ensemble have approximately the same velocities. As far as the atoms with the initial velocity of $v_{0}=-25 \mathrm{~m} / \mathrm{s}$ are concerned, the situation is completely different. In this case the corresponding curve in Fig. 4, a passes much lower than the curves corresponding to $v_{0}=0, \pm 10 \mathrm{~m} / \mathrm{s}$. The reason is obvious - in the ensemble of atoms there is a significant variation of $k \Delta v$ Doppler frequency shifts relative to the average Doppler shift $k \bar{v}$ (see the corresponding curve in Fig. 4, b), and this dramatically affects the interaction of atoms with the field. In this case, it is manifested by a marked decrease in the average acceleration of atoms.

Note that the oscillations on all three curves with $v_{0}=0, \pm 10 \mathrm{~m} / \mathrm{s}$ in Fig. 4, $a$ for $\bar{v}>20 \mathrm{~m} / \mathrm{s}$, when the average acceleration of atoms is close to zero, do not match. This suggests that the oscillations are due to fluctuations when averaged over a relatively small number of atoms.

At the beginning of the interaction of atoms with the field, the standard deviation of the velocity from the mean value changes approximately according to the law $\Delta v \propto \sqrt{2 D_{v} t}$ (see Fig. 3). Over time, the dependence of $\Delta v$ on time (as well as on the average velocity $\bar{v}$ of atoms, as far as $\bar{v}$ grows monotonically with time) ceases to be monotonic: are observed as areas of growth, and the decline of $\Delta v$ (see Fig. 4, $b$ ).

To understand the nonmonotonic change of $\Delta v$ with a change of $\bar{v}$, we must take into account two factors that determine it. On the one hand, it is a process of momentum diffusion, which leads to a diffusion-like change of $\Delta v$ with time, as can be seen in Fig. 3 , On the other hand, the dependence of the average acceleration $a$ on the average velocity $\bar{v}$ of an atom can both increase and decrease the value of $\Delta v$. Indeed, let us consider the case $d a / d \bar{v}<0$. It is plausible to assume that the acceleration $a_{1}$ of one atom or group of atoms with velocity $v_{1}$ also decreases with increasing velocity, $d a_{1} / d v_{1}<0$. The accuracy of this statement becomes greater if the standard deviation $\Delta v$ of the velocity of atoms 
in the ensemble from its mean value becomes smaller. In this case, the acceleration of atoms with a lower velocity is greater than the acceleration of atoms with a higher velocity. As a result, the velocity distribution of atoms narrows to a limit that is established as a result of the dynamic equilibrium of the distribution narrowing process by reducing the acceleration of atoms with increasing velocity and the momentum diffusion process that makes broader this distribution. A well-known analog of this phenomenon is the Doppler cooling of atoms [8], with the difference that instead of grouping atoms at zero velocity in the field of a standing monochromatic wave, we have the grouping of atoms near the average velocity of the ensemble of atoms $\bar{v}$ in the field of counter-propagating bichromatic waves in the case of $d a / d \bar{v}<0$. Similar considerations show that in the case of $d a / d \bar{v}>0$ there should be an expansion of the distribution of atoms by momentum. Besides that, in this case, the dependence of the acceleration of atoms on their velocity changes $\Delta v$ in the same direction as the momentum diffusion. The described phenomena are clearly seen in Fig. 4 ,

Now, understanding the physical reason for the nonmonotonic dependence of $\Delta v$ on $\bar{v}$, we will dwell on some details of the dependencies shown in Fig. 4. First, we note the shift of the maximum of $\Delta v(\bar{v})$ relative to $a(\bar{v})$ towards the larger $\bar{v}$, which is well visible for the curve corresponding to initial velocity $v_{0}=-25 \mathrm{~m} / \mathrm{s}$. The reason for this shift is obvious. The derivative $d a / d \bar{v}$ near the maximum of the dependence $a(\bar{v})$ is too small to compensate the increase in $\Delta v$ due to the momentum diffusion. The small value of the derivative $d a / d \bar{v}$ in the range from $20 \mathrm{~m} / \mathrm{s}$ to $25 \mathrm{~m} / \mathrm{s}$, insufficient to compensate the momentum diffusion, is also the reason for the growth of $\Delta v$ for curves with $v_{0}=0 \mathrm{~m} / \mathrm{s}, v_{0}=5 \mathrm{~m} / \mathrm{s}$ and $v_{0}=10 \mathrm{~m} / \mathrm{s}$ in Fig. 4. $b$. For the curve with $v_{0}=-25 \mathrm{~m} / \mathrm{s}$, the derivative $d a / d \bar{v}$ in the interval from $5 \mathrm{~m} / \mathrm{s}$ to $30 \mathrm{~m} / \mathrm{s}$ is large enough to monotonically reduce the standard deviation of the velocities of atoms from the mean value, and no local maxima to the right of $v_{0}=5 \mathrm{~m} / \mathrm{s}$ are observed.

Similar behavior of $\Delta v$ for ${ }^{23} \mathrm{Na}$ atoms occurs also for atoms ${ }^{133} \mathrm{Cs}$. Fig. 5 shows the dependence of the average acceleration $a=d \bar{v} / d t$ on the average velocity of $\bar{v}$ atoms, calculated after smoothing $\bar{v}(t)$ by the program GNUPLOT with smoothing parameter $1(a)$ and the dependence of the standard deviation of the velocity $\Delta v$ on its mean value $\bar{v}(b)$. The calculations were performed for the same values of $\Omega_{0} / \gamma, \Omega / \gamma$, and $\varphi$, as well as for sodium atoms in Fig. 4. Again, as in Fig. 目, we see a decrease in $\Delta v$ with decreasing of the average acceleration of atoms (see the range of $\bar{v}>8 \mathrm{~m} / \mathrm{s}$ ). We carried out the calculations for different implementations of sequences of random numbers (see Section 3) 

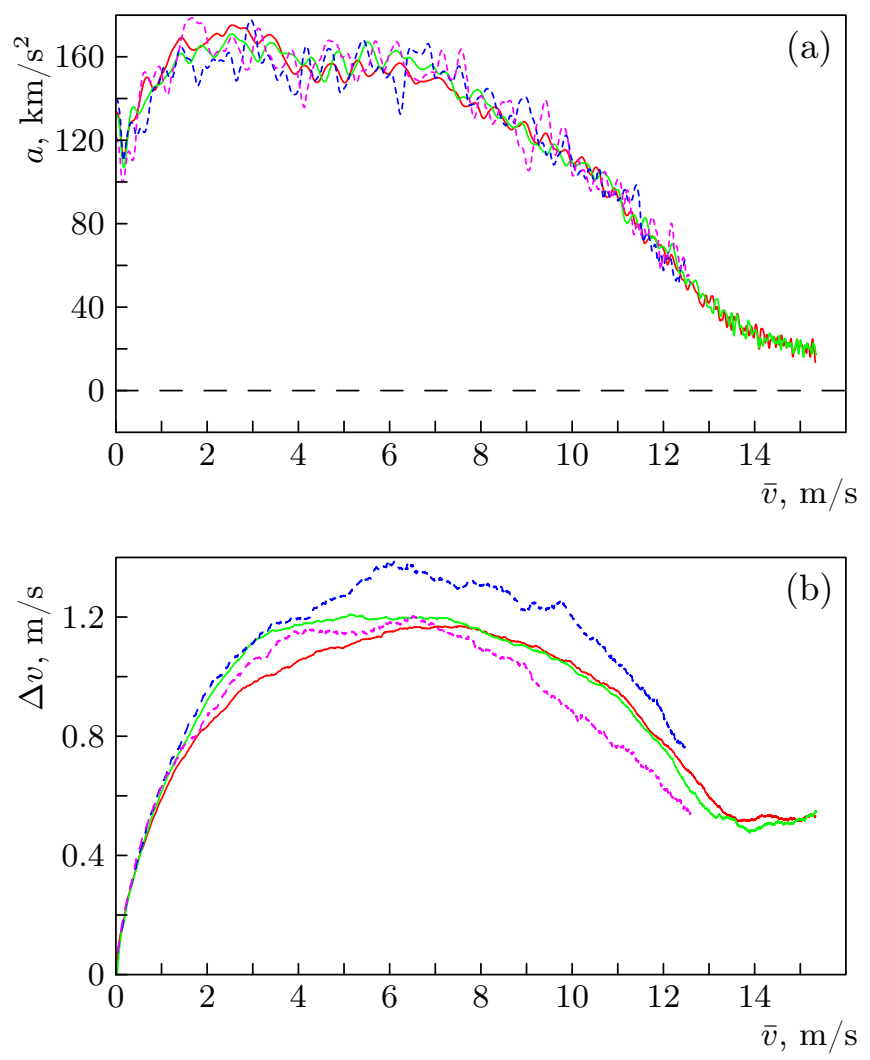

FIG. 5. Dependence of the average acceleration $a=d \bar{v} / d t$ on the average velocity of $\bar{v}$ cesium atoms, calculated after smoothing $\bar{v}(t)$ by the program Gnuplot (a) and dependence of the standard deviation of the velocity $\Delta v$ on its average value $\bar{v}$ (b) for 500 (solid curve) and 100 (dashed curve) cesium atoms in the field of counter-propagating bichromatic waves for different implementations of sequences of random numbers. Parameters are: $\Omega_{0}=2 \pi \times 63.44 \mathrm{MHz} ; \Omega=2 \pi \times 51.8 \mathrm{MHz}$. The initial velocity of atoms $v_{0}=0$, the phase difference of the opposing waves $\varphi=p i / 4$. The calculations were performed for the atomic motion time of $100 \mu \mathrm{s}$ in the case of 100 atoms and 200 $\mu \mathrm{s}$ in the case of 500 atoms

and different numbers of atoms in the ensemble. These allow us to estimate the accuracy of the calculations. Comparison of the curves in Fig. 5, $a$ allows us to talk about the accuracy of calculations of $a$ at the level of $7-10 \%$ for 500 atoms and up to $20 \%$ for 100 atoms. Comparison of the curves in Fig. 15, $b$ allows us to estimate the accuracy of the calculations of $\Delta v$ at the level $15 \%$ for 500 atoms and $25 \%$ for 100 atoms.

Since the velocity of atoms changes continuously with time, the momentum diffusion coefficient also changes with time. To completely exclude the effect of a change in velocity on 
the momentum diffusion coefficient, in the next section we consider the change in momentum of an atom with time for the "heavy atom" model when the change in atomic velocity can be neglected. In addition, this consideration will allow us to compare the results of the calculation of the light pressure force exerted on an atom in the quasiclassical theory [1, 6, 6 ] with the results of the quantum mechanical theory. Since in quasi-classical theory the atom is considered as a material point, and in our calculations, the atom at the beginning of interaction with the field is considered as a monochromatic wave, consistency of the results obtained from these opposite assumptions is important for the confident application of the quasi-classical approach in the cases where we are interested only in the light pressure force exerted on an atom.

\section{B. Heavy atoms in the field of counter-propagating bichromatic waves. Light} pressure force and momentum variance

We simulate the motion of "heavy" atoms in the field of the counter-propagating waves by the procedure described in Sec. IV] and use Eqs. (34), (35), which describe the time evolution of the probability amplitudes $b_{g, n}, b_{e, n}$. Calculating the change of the average momentum of the atoms during the time $\sim 100 \gamma^{-1}$, much greater than the time of the transient processes at the beginning of the interaction of atoms with the field $\left(\sim 10 \gamma^{-1}\right)$, we find the average force exerted on an atom. In addition, we calculate the light pressure force by the density matrix method according to the theoretical work [1]. As can be seen from Fig. 6, the calculations by both methods are quite close, despite the relatively low accuracy of the calculation by the Monte Carlo method for an ensemble of 100-1000 atoms.

Now let's consider the change of the standard deviation $\Delta p$ of the momentum from its average value (square root of the variance of the momentum) on time. In the previous section, it was shown that at the beginning of the interaction of the atom with the field $\Delta p \sim \sqrt{t}$ due to momentum diffusion. Further, because of the change of the atomic velocity, the law describing the dependence of $\Delta p$ on time changes. Here we consider similar dependencies for the "heavy atom" model. An example of the numerical simulations is shown in Fig. 7 for zero initial velocity of atoms. As we can see, for a standing monochromatic wave (curve 1) $\Delta p \sim \sqrt{t}$, which indicates the diffusion nature of the blurring of the distribution of atoms by momentum. The momentum diffusion coefficient is $D=74.3 D_{r}$. Even a relatively small 

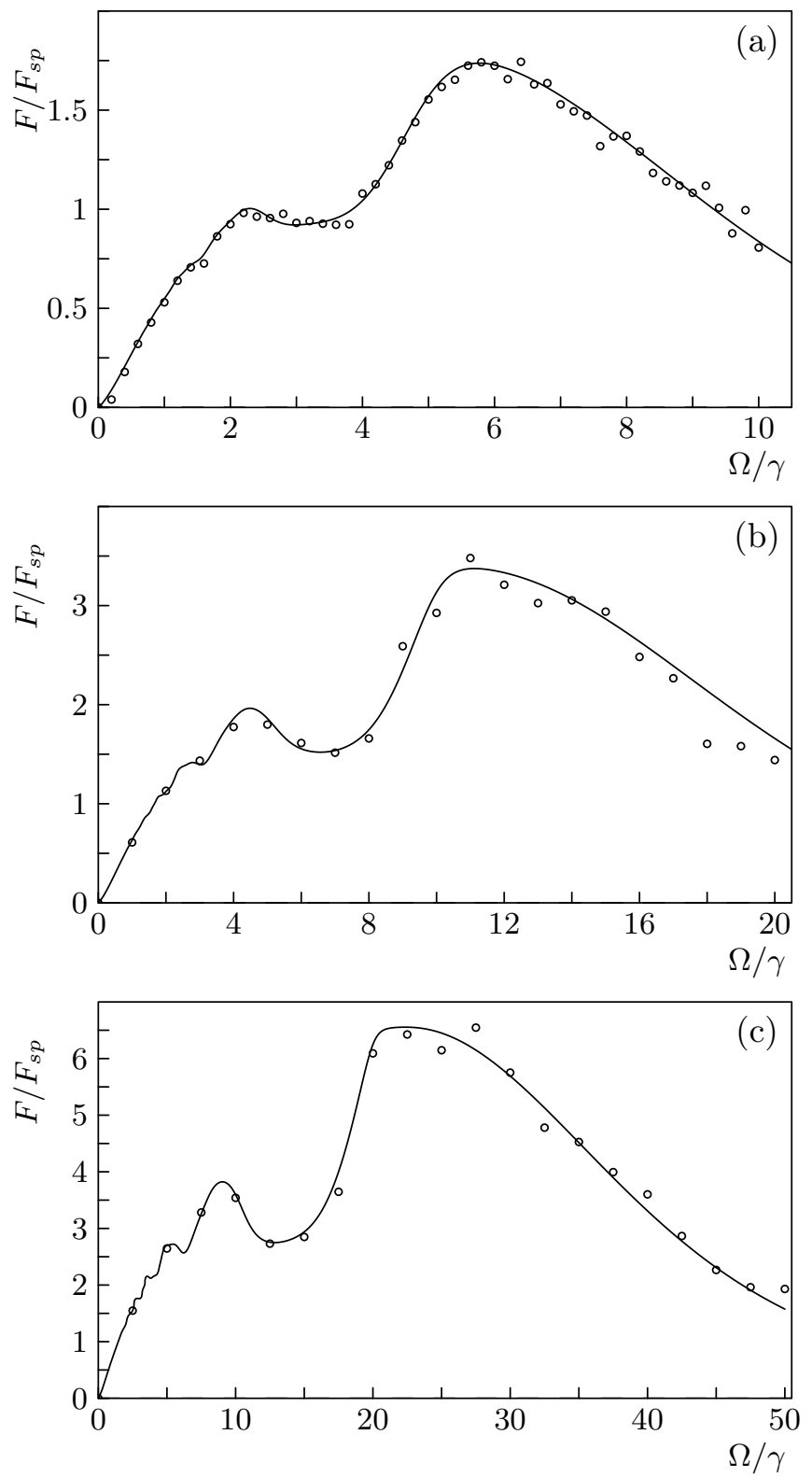

FIG. 6. The dependence of the light pressure force exerted on an atom in units of $F_{s p}$ on $\Omega / \gamma$. Solid curves - calculation based on the results of work [1], circles — calculation by the Monte Carlo wave function method. (a) $-\Omega_{0} / \gamma=6.124,1000$ atoms; (b) $-\Omega_{0} / \gamma=12.25$, 100 atoms; (c) $-\Omega_{0} / \gamma=24.5,1000$ atoms. The initial velocity of the atoms is $v_{0}=0$. Phase difference of the counter-propagating waves is $\varphi=\pi / 4$

difference of frequencies between the components of bichromatic waves, $\Omega=0.2 \gamma$, leads to a significant change in the dependence of $\Delta p(t)$. As can be seen, for the initial moments of time, $t<4 \gamma^{-1}$, curves 1 and 2 almost coincide, later on, curve 2 oscillations are observed, 


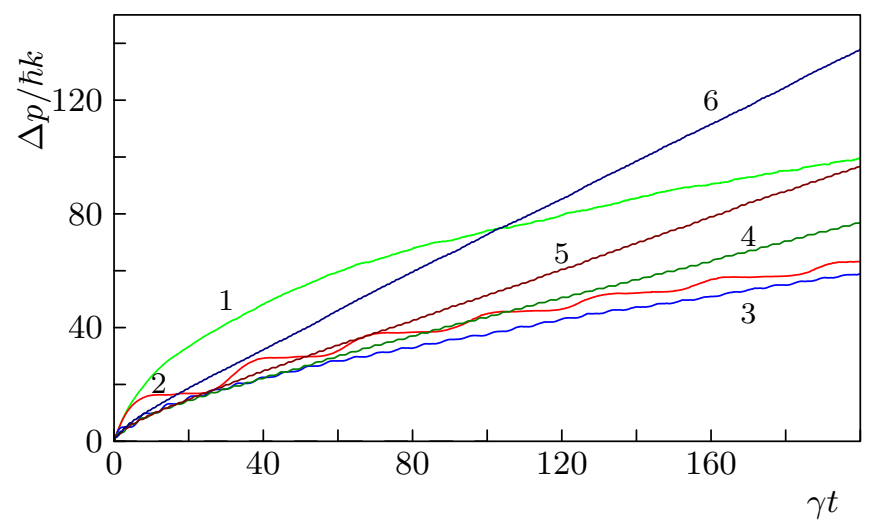

FIG. 7. Time dependence of the standard deviation $\Delta p$ of the atom's momentum from its average value for for 1000 atoms and $\Omega_{0} / \gamma=6.124$. Parameters are: $1-\Omega=0 ; 2-\Omega=0.2 \gamma ; 3-\Omega=\gamma$; $4-\Omega=2 \gamma, 5-\Omega=3 \gamma, 6-\Omega=5 \gamma$. The initial velocity of the atoms is $v_{0}=0$. Phase difference of the counter-propagating waves is $\varphi=\pi / 4$

the period of which is $2 \pi / \Omega$. The momentum diffusion coefficient determined from the approximation of curve 2 by the root dependence is equal to $D=27.6 D_{r}$, i.e. 2.7 times less than for a standing monochromatic wave (curve 1). As $\Omega$ increases, the amplitude of the oscillations and their period decrease (curves 3-6), and the root dependence of smoothed curves $3-6$ of $\Delta p$ on time becomes almost linear. Curve 2 is similar to the root dependence (taking into account the smoothing of oscillations), curve 3 at the beginning is also close to the graph of the square root, but closer to the end of the abscissa axis is more like a graph of a linear function, and curve 6 is a straight line on the almost whole abscissa axis interval. Thus, for small values of $\Omega$ we have the diffusion of atoms in the momentum space, and for greater $\Omega$ the dependence $\Delta p(t)$ corresponds to the scattering of atoms in a certain range of angles. In addition, with the transition from a monochromatic field to a bichromatic one, even at $\Omega \ll \gamma$ the momentum diffusion coefficient decreases sharply.

Comparing Fig. 7 and Fig. 3, we see an obvious contradiction: in Fig. 3 the diffusion-like dependence of the standard deviation $\Delta v$ of the velocity of atoms on its average value for $\Omega \sim \Omega_{0}$ is clearly visible, at the same time in Fig. 7 analogous dependence for the standard deviation of the atom's momentum from its average value is close to linear. Since the dependences shown in Fig. 3 are calculated taking into account the change in the velocity of atoms over time, we should investigate the dependence of $\Delta p$ on time for a nonzero initial velocity of a "heavy" atom. 
Fig. 8 shows the time dependence of the standard deviation $\Delta p$ of the atom's momentum from its average value for $\Omega_{0}=6.124 \gamma, \Omega=5 \gamma$ (optimal ratio of $\Omega$ and $\Omega_{0}$ according to [6]). As can be seen, the case $v_{0}=0$ is special: only in this case the dependence of $\Delta p$ on $t$ is

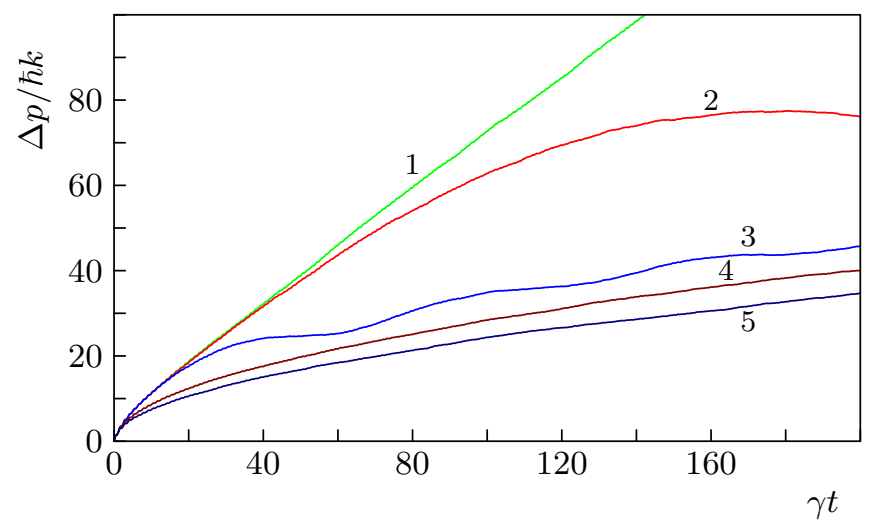

FIG. 8. Time dependence of the standard deviation $\Delta p$ of the atom's momentum from its average value for 1000 atoms and $\Omega_{0}=6.124 \gamma, \Omega=5 \gamma$. Parameters are: $1-k v_{0}=0,2-k v_{0}=0.01 \gamma$, $3-k v_{0}=0.05 \gamma, 4-k v_{0}=\gamma, 5-k v_{0}=2 \gamma$. Phase difference of the counter-propagating waves is $\varphi=\pi / 4$

linear for $\gamma t>10$. If the initial velocity is nonzero, oscillations with frequency $k v_{0}$ appear on the specified dependence. The dependence $\Delta p(t)$ for $v_{0}=0$ can be considered as oscillating with zero frequency. At the beginning of the interaction with the field, at $\gamma t \ll 1$, all the graphs almost coincide with the curve constructed for $v_{0}=0$. As can be seen, for $k v_{0} \geq \gamma$, besides the initial moments of time, when the transients associated with the spontaneous emission play a significant role, the dependence of $\Delta p$ on time is described by the law of quadratic root. Thus, for real atoms of finite mass, in which the velocity changes during interaction with the field, momentum diffusion should be observed, as shown in Fig. 3 .

Momentum diffusion coefficients, found by approximating the curves shown in Fig. 8 with the square root law using the method of least squares, are: $D=14.9 D_{r}$ (curve 3 ), $D=11.6 D_{r}$ (curve 4$), D=8.4 D_{r}$ (curve 5$)$.

Oscillations with a period corresponding to the frequency difference between the monochromatic components of the bichromatic waves are also observed in the corresponding to Fig. 7 time dependencies of the average momentum $\bar{p}$ of atoms (see Fig. 9). As can be seen from Fig. 9, curves 4 and 5 almost coincide, which indicates the same light pressure force exerted on the atoms for $\Omega=2 \gamma$ and $\Omega=3 \gamma$, in conformity with Fig. 6, $a$. 


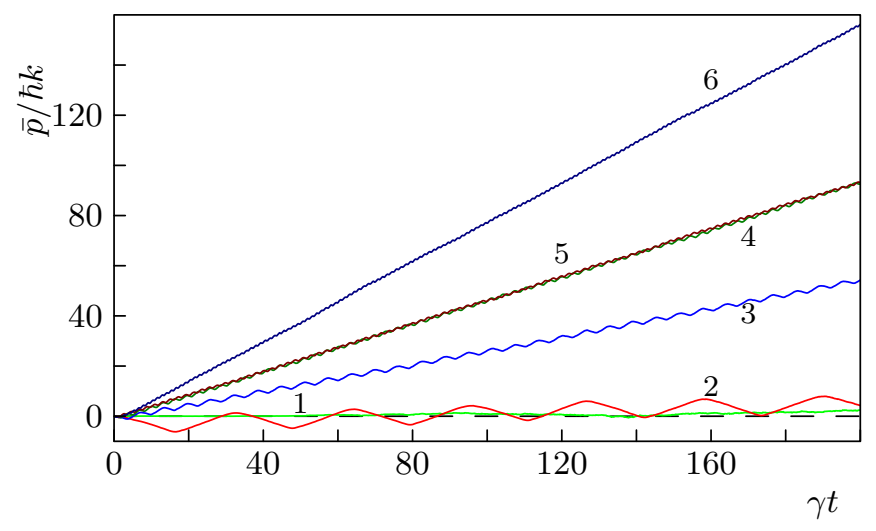

FIG. 9. Time dependence of the average momentum $\bar{p}$ of an atom for 1000 atoms and $\Omega_{0} / \gamma=$ 6.124. Parameters are: $1-\Omega=0,2-\Omega=0.2 \gamma, 3-\Omega=\gamma, 4-\Omega=2 \gamma, 5-\Omega=3 \gamma, 6-\Omega=5 \gamma$. The initial velocity of the atoms $v_{0}=0$. Phase difference of counter waves $\varphi=\pi / 4$

Fig. 8 shows that the width of the atomic momentum distribution at the beginning of the atom-field interaction for zero initial velocity of atoms linearly depends on time. When applying the calculations to real atoms, it should be borne in mind that even a slight change in the velocity of atoms is enough to change the linear law to nonlinear, which is well described by the law of the square root at $k v_{0}=0.05 \gamma$.

The dependencies of the momentum diffusion coefficient and the average light pressure force on $\Omega$ for $k v_{0}=0.1 \gamma$ and $k v_{0}=0.2 \gamma$ are shown in Fig. 10, As can be seen in Fig. 10, $a$, for $\Omega=20 \gamma=\sqrt{2 / 3} \Omega_{0}$ the light pressure force for both values of $k v_{0}$ is almost the same. According to the work [6], this should be the case with the optimal ratio of $\Omega_{0} / \Omega=\sqrt{3 / 2}$, when the light pressure force changes a little with the speed of the atom near the maximum value of force. The momentum diffusion coefficient as a function of $\Omega$ (see Fig. 10, $b$ ) reaches a local maximum at this point. The absolute maximum of the momentum diffusion coefficient $\left(D / D_{r}=1371, D / D_{r}=1259\right.$ for $k v_{0} / \gamma=0.1$ and $k v_{0} / \gamma=0.2$ respectively $)$ is reached only at $\Omega=0$ when the counter-propagating bichromatic waves become monochromatic. Thus, the momentum diffusion coefficient under the condition of the optimal ratio of $\Omega$ and $\Omega_{0}$ is six times smaller than the momentum diffusion coefficient in the counter-propagating monochromatic waves, in which the counter-propagating bichromatic waves degenerate at $\Omega=0$.

Besides the case of zero initial velocity of atoms (recall that in the approximation of a heavy atom we neglect the change of velocity, assuming that only the momentum of the 

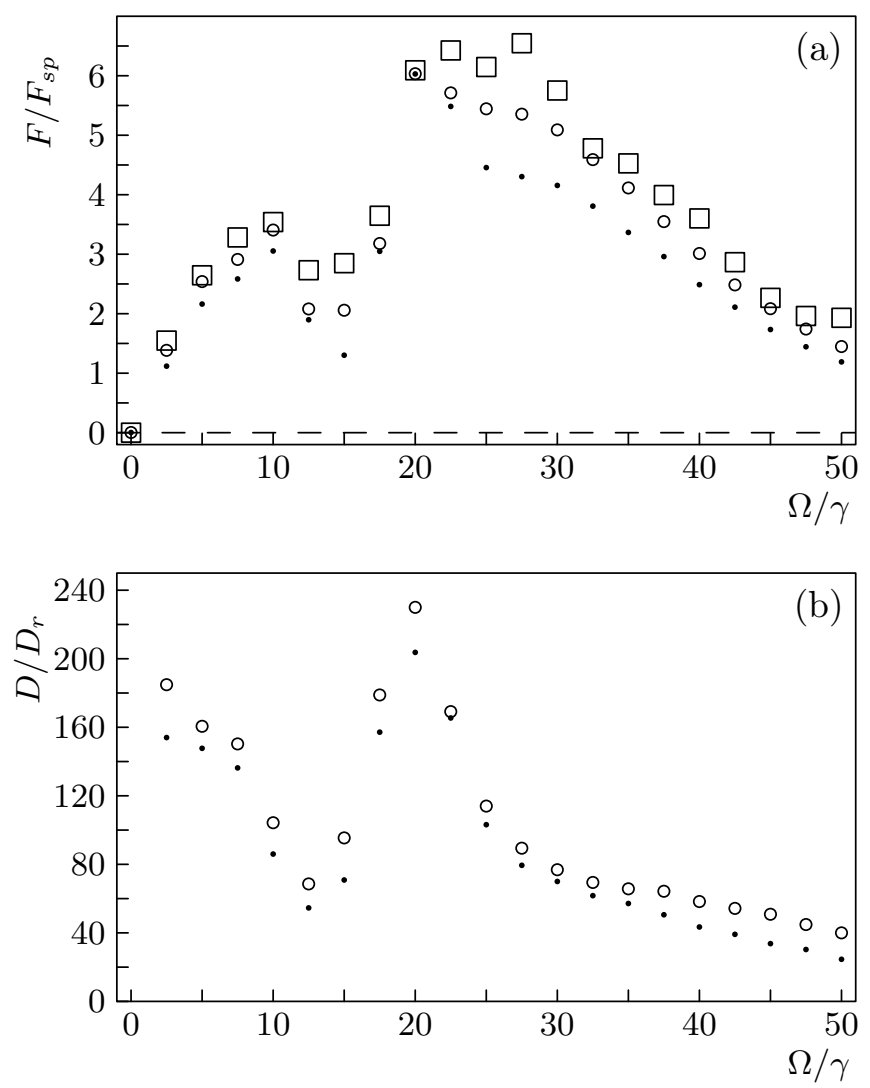

FIG. 10. Dependencies of the light pressure force $F$ in units of $F_{s p}(a)$ and the momentum diffusion coefficient $(b)$ on $\Omega / \gamma$ for different initial velocities $v_{0}$ of atoms for $\Omega_{0} / \gamma=24.5$. Averaging per 1000 atoms, the phase difference of the counter-propagating waves is $\varphi=\pi / 4$. Rings $-k v_{0} / \gamma=0.1$, circles $-k v_{0} / \gamma=0.2$, squares $-v_{0}=0$. For the case $\Omega=0$ we have (not shown) $D / D_{r}=1371$ for $k v_{0} / \gamma=0.1$ and $D / D_{r}=1259$ for $k v_{0} / \gamma=0.2$

atom changes with time), there are other values of the initial velocity of atoms at which the dependence $\Delta p(t)$ is quite well described by linear law. The transition from scattering to diffusion and vice versa with a change in the initial velocity of the atom is illustrated in Fig. 11, It shows the time dependence of $\Delta p^{2}(t)$, which in the case of momentum diffusion is close to linear. Fig. 11 also shows the time dependence of the varying part $\bar{p}$ of the total average momentum of atoms $m v_{0}+\bar{p}$, which we will call as "the average momentum of the atom" to simplify the terminology. It is noteworthy that while curves 1 and 2 in Fig. 11, $a$ are very close, the corresponding curves in Fig. 11, $b$ are close only at the beginning of the interaction of the atoms with the field. Curve 1 in Fig. 11, $b$ is a parabola, it corresponds to the linear change of $\Delta p$ with time. Thus, in the case of $v_{0}=0$ we have the scattering 

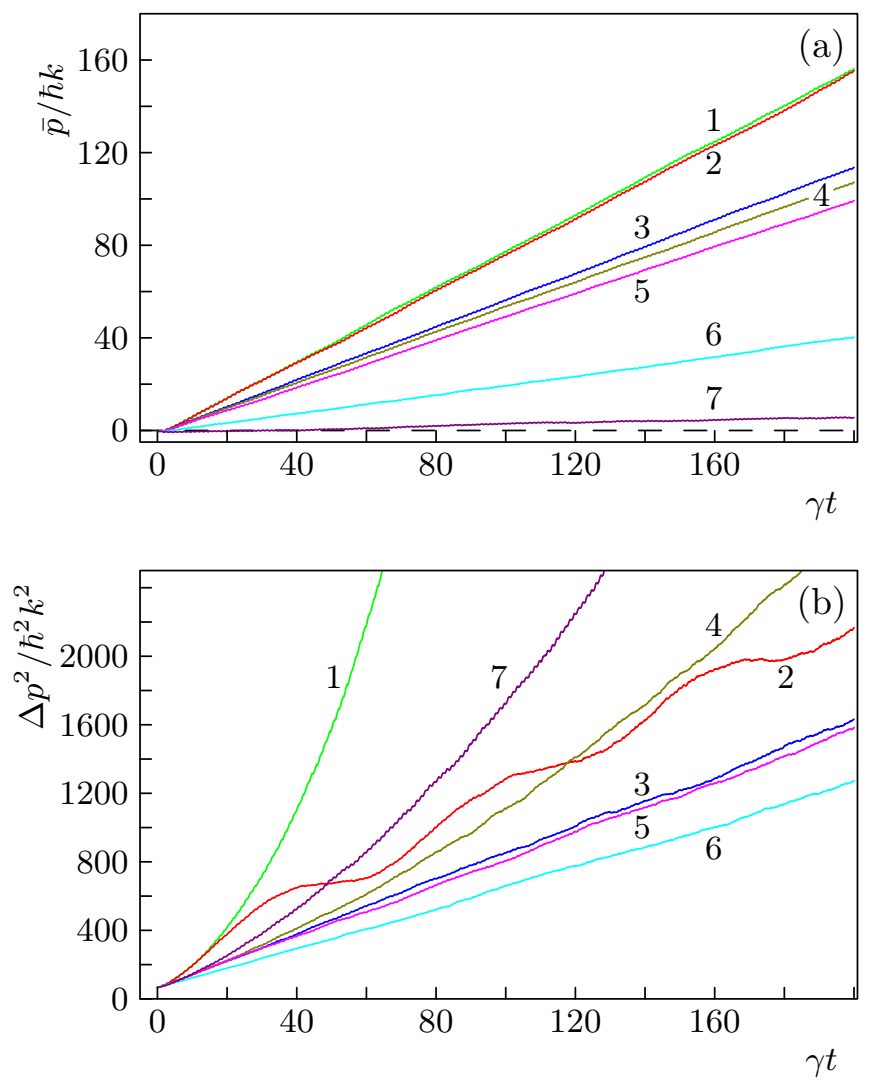

FIG. 11. Time dependencies of the average momentum $\bar{p}$ of atoms $(a)$ and the average square of the deviation of the momentum from the average value $\Delta p^{2}(b)$. Parameters are: $\Omega_{0} / \gamma=6.124$, $\Omega / \gamma=5$. Averaging per 1000 atoms. The phase difference of counter waves is $\varphi=\pi / 4$. $1-$ $v_{0}=0,2-k v_{0} / \gamma=0.05,3-k v_{0} / \gamma=1.2,4-k v_{0} / \gamma=1.25,5-k v_{0} / \gamma=1.3,6-k v 0 / \gamma=2,7-$ $k v_{0} / \gamma=2.5$

of atoms. Even when the initial velocity of atoms becomes relatively small (see curve 2 in Fig. 11, b), the time dependence of $\Delta p^{2}$ dramatically changes. It becomes almost, with accuracy to small oscillations, a linear function, and $\Delta(t)$ is approximately described by the function $\Delta p=\sqrt{2 D t}$. In this case, we can talk about the momentum diffusion, which is described by the momentum diffusion coefficient $D$. As the initial velocity increases, $\Delta p(t)$ is approximately described by the diffusion law until the velocity becomes $v_{0}=\Omega /(4 k)$. We see that the time dependence of the average momentum of atoms at $v_{0}=\Omega /(4 k)$ (curve 4 ) differs a little from similar dependences at a small change in velocity in one direction or another (curves 3 and 5); the corresponding dependencies of $\Delta p^{2}$ on time differ radically: curve 4 is a parabola (scattering of atoms), curves 3 and 5 - are graphs of linear functions 
(momentum diffusion). Probably the transition to scattering at the initial velocity of atoms equals $v_{0}=\Omega /(4 k)$ is associated with Doppleron resonance [19, 20], which in this case is due to a four-photon process: the absorption of two quanta of light from the monochromatic component of the counter-propagating bichromatic wave of frequencies $\omega+\frac{1}{2} \Omega+k v_{0}$ and $\omega-\frac{1}{2} \Omega+k v_{0}$ and the emission of two quanta of light into a monochromatic component of a concomitant bichromatic wave of frequency $\omega+\frac{1}{2} \Omega-k v_{0}$ or a four-photon process with the absorption of two quanta from the monochromatic component of frequency $\omega-\frac{1}{2} \Omega+k v_{0}$ from the counter-propagating wave and the emission of quanta with frequencies $\omega-\frac{1}{2} \Omega-k v_{0}$, $\omega+\frac{1}{2} \Omega-k v_{0}$ in monochromatic components of the concomitant bichromatic wave. With increasing $v_{0}$ we again fall into the region of momentum diffusion (see curve 6). Other theoretically possible Doppleron resonances (for example, at $v_{0}=\Omega /(3 k)$ ) at the parameters of interaction of atoms with the field corresponding to Fig. 11 were not registred, until reaching the velocity $v_{0}=\Omega /(2 k$ ) (curve 7 ), where the time dependence of $\Delta p$ corresponds to the scattering of atoms. Note that this velocity corresponds to the single-photon resonance of waves with frequencies $\omega-\frac{1}{2} \Omega+k v_{0}$ and $\omega+\frac{1}{2} \Omega-k v_{0}$ and reducing the force of light pressure almost to zero [1].

Fig. 12 shows the dependence of the light pressure force $F$ exerted on atoms in units of $F_{s p}$ and the square root of the momentum diffusion coefficient $\sqrt{D}$ in units of $\sqrt{D_{r}}$ on the difference of frequencies of the monochromatic components of the counter-propagating bichromatic waves in units of $\gamma$ provided that the optimal conditions of the atom-field interaction are fulfilled: $\Omega_{0}=\sqrt{3 / 2} \Omega, \varphi=\pi / 4$. The values of the light pressure force $F=\bar{p} / t$ and the pulse diffusion coefficient $D=\Delta p^{2} /(2 t)$ were calculated by the method of least squares from the dependencies $\bar{p}(t)$ and $\Delta p^{2}(t)$ which were calculated for an ensemble of 1000 atoms in the time interval from 0 to 100/ $\gamma$. Using the dependence shown in Fig. 12 , $b$, we find for the case $k v_{0}=0.1 \gamma$ :

$$
D \approx 0.6 D_{r} \frac{\Omega^{2}}{\gamma^{2}}
$$

This is about twice as much, as gives the Eq. (47).

As expected, according to the theoretical work [6], the force exerted on an atom under optimal conditions of the atom-field interaction depends linearly on $\Omega$. According to our calculations, the square root of the pulse diffusion coefficient also depends linearly on $\Omega$. 

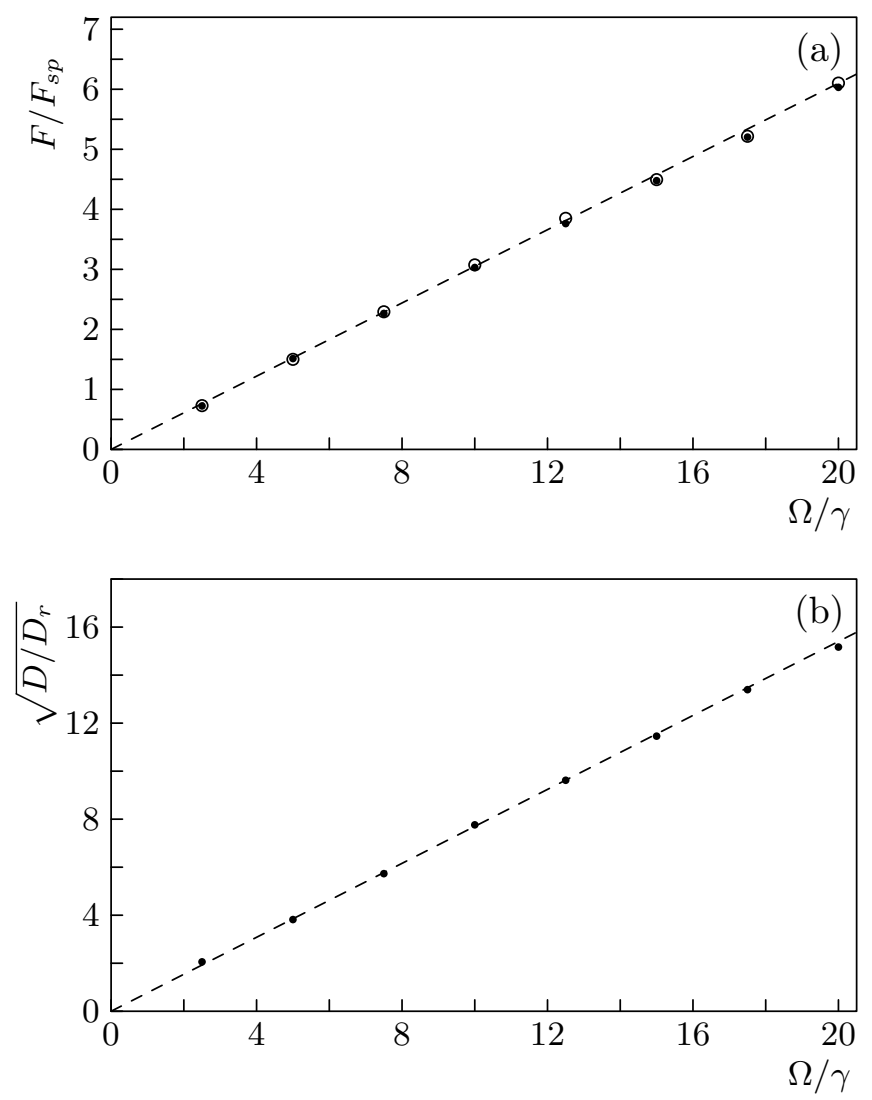

FIG. 12. Dependence of the light pressure force exerted on atoms in units of $F_{s p}(a)$ and the square root of the coefficient of momentum diffusion $\sqrt{D}$ in units of $\sqrt{D_{r}}(b)$ on the frequency difference of the components of the counter-propagating bichromatic wave in units of $\gamma$ provided that the Rabi frequency $\Omega_{0}=\sqrt{3 / 2} \Omega$ and $\varphi=\pi / 4$. Averaging per 1000 atoms. Rings correspond to $v_{0}=0$, circles correspond to $k v_{0}=0.1 \gamma$

This means that the ratio $\sqrt{D} / F$, and, as a result

$$
\frac{\Delta p}{\bar{p}}=\frac{\sqrt{2 D t}}{F t} \approx 2.5 \frac{\sqrt{2 D_{r}}}{F_{s p} \sqrt{t}} \approx \frac{4.2}{\sqrt{\gamma t}}
$$

does not depend on $\Omega$. Since $\Omega_{0}=\sqrt{\frac{3}{2}} \Omega$, the momentum diffusion coefficient under optimal conditions of the atom-field interaction is proportional to $\Omega_{0}^{2}$, i.e. the intensity of the laser radiation.

Fig. 13 shows the dependence of the light pressure force exerted on atoms in the field of counter-propagating bichromatic waves and the momentum diffusion coefficient $D$ on the atomic velocity for the optimal ratio $\Omega_{0} / \Omega=\sqrt{3 / 2}$ and $\varphi=\pi / 4$, which were found from the time dependence of the change of the average momentum and the average square 

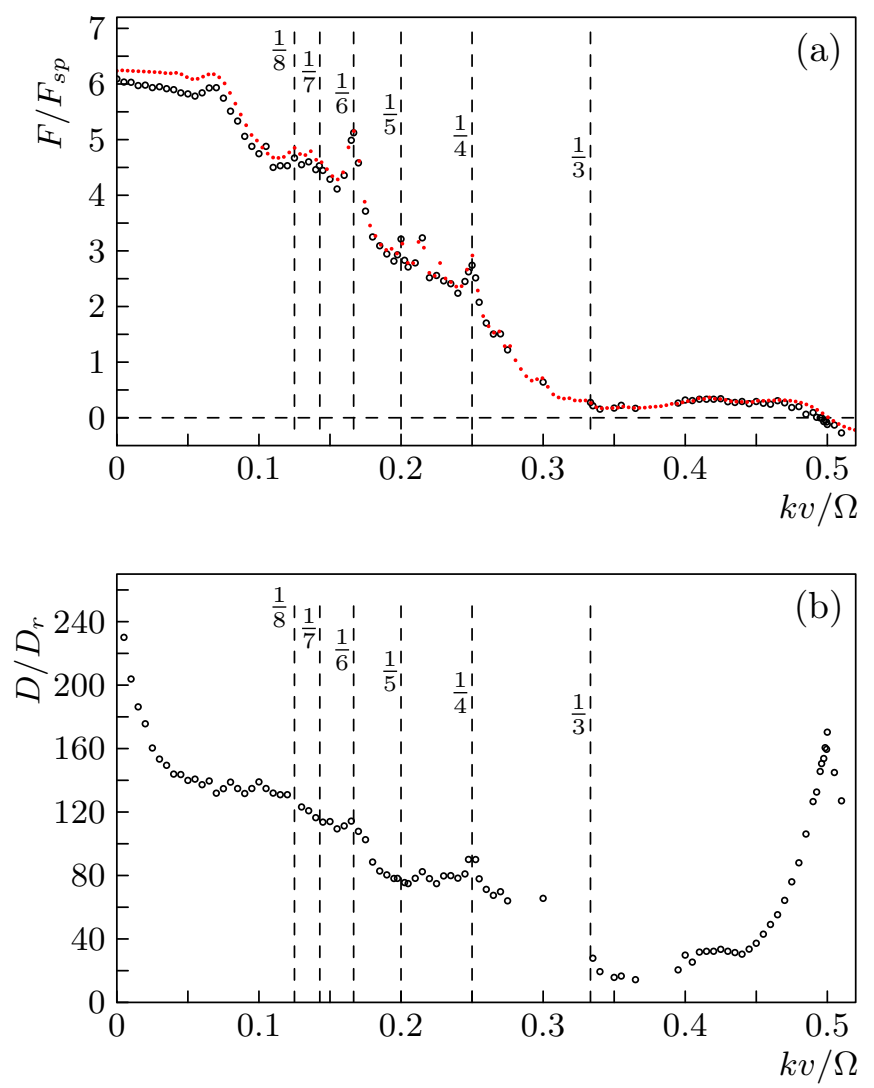

FIG. 13. The dependences of the light pressure force $F$ exerted on atoms in units of $F_{s p}(a)$ and the momentum diffusion coefficient $D$ in units of $D_{r}(b)$ on the atomic velocity for $\Omega_{0}=\sqrt{3 / 2} \Omega$, $\Omega=20 \gamma$ and $\varphi=\pi / 4$. Averaging per 1000 atoms. Rings are the results of calculation by the Monte Carlo wave function method, the solid curve is the results of calculations by the density matrix equation

momentum during time $t=100 / \gamma$. The results of the calculation of the light pressure force on atoms by the equations for the density matrix, similar to the works [1, 5], are also presented. The dependencies of the force on velocity, calculated by both methods, almost coincide. Some differences can be explained by different descriptions of the atom: in our calculations, the atom at the beginning of the interaction with the field is a plane wave, in the calculations by the equations for the density matrix the atom is a material point. It is noteworthy that the coefficient of momentum diffusion with increasing velocity $v$ of atoms at $k v<0.05 \gamma$ decreases rather quickly, almost twice when velocity changes from $v=0.005 \Omega / k$ to $v=0.05 \Omega / k$, while the light pressure force remains almost unchanged in this velocity range. Note that points on the graph correspond to the velocity $v$ of different ensembles of atoms, which for each ensemble in the approximation of "heavy" atom during its movement 
remains unchanged and equal to the initial velocity $v=v_{0}$. At velocities corresponding to Doppleron resonances (denoted by vertical dotted lines), the momentum diffusion coefficient could not be calculated because the time dependence of the variance of the momentum is not described by the law $\Delta p^{2}=2 D t$.

The results of this section are valid in the approximation of a heavy atom when the change of its velocity during the atom-field interaction is neglected. For real atoms, the transition from scattering to momentum diffusion and vice versa occurs in a short time and is not noticeable in Fig. 1 and Fig. 3, perhaps due to insufficient accuracy of our calculations by the Monte Carlo method.

\section{CONCLUSIONS}

The calculation of the light pressure force exerted on atoms in the field of counterpropagating bichromatic waves based on the quantum mechanical description of the mechanical motion of atoms agrees well with the previous studies of the light pressure based on the classical description of mechanical motion. That is an additional substantiation of the classical approach to the problems of the motion of atoms in the field of the counterpropagating modulated waves.

The standard deviation of the velocity from its average value may increase or decrease with time. This correlates with the increase or decrease of the average acceleration of atoms and is associated with both the momentum diffusion of atoms and bunching or the antibunching of atoms around the average velocity of the atomic ensemble.

We calculated the momentum diffusion of atoms in the field of counter-propagating bichromatic waves both for ideal cases of "heavy" atoms and for sodium atoms. We have shown that the analogy between the interaction of atoms with the field of counterpropagating bichromatic waves and the interaction of atoms with the field of counterpropagating $\pi$-pulses can be used for a rough estimation of the momentum diffusion coefficient of the atoms in the field of the counter-propagating bichromatic waves.

Depending on the parameters of the interaction of atoms with the field, the time dependence of the momentum variance corresponds to either scattering of atoms (square root of the momentum variance is proportional to time) or diffusion of atoms (the momentum variance is proportional to time). Momentum diffusion changes to the scattering of atoms 
when the velocity of atoms approaches Doppleron resonances.

It is shown that under the conditions of optimal parameters of the interaction of atoms with the field, the coefficient of momentum diffusion is proportional to the intensity of laser radiation. Under these conditions, the ratio of the root mean square deviation of atomic momentum from its average value to the average atomic momentum does not depend on $\Omega$.

The publication contains the results of research in the frame of grant support of the target complex program of fundamental research of NAS of Ukraine "Fundamental problems of creation of new nanomaterials and nanotechnologies", contract No 3/19-N, as well as on the theme V-185.

[1] V. S. Voitsekhovich, M. V. Danileiko, A. M. Negriiko, V. I. Romanenko, and L. P. Yatsenko, Sov. Phys. Tech. Phys. 33, 690 (1988).

[2] V. G. Minogin and V. S. Letokhov, Laser Light Pressure on Atoms (Gordon and Breach: New York, 1987).

[3] H. J. Metcalf and P. van der Stratten, Laser Cooling and Trapping (Springer-Verlag: New York, Berlin, Heidelberg, 1999).

[4] V. S. Voŭtsekhovich, M. V. Danileı̌ko, A. N. Negriı̌ko, V. I. Romanenko, and L. P. Yatsenko, JETP Lett. 49, 161 (1989).

[5] J. Söding, R. Grimm, Y. Ovchinnikov, P. Bouyer, and C. Salomon, Phys. Rev. Lett. 78, 1420 (1997).

[6] L. Yatsenko and H. Metcalf, Phys. Rev. A 70, 063402 (2004).

[7] L. Podlecki, R. D. Glover, J. Martin, and T. Bastin, J. Opt. Soc. Am. B 35, 127 (2018).

[8] H. Metcalf, Rev. Mod. Phys. 89, 041001 (2017).

[9] K. Berg-Sørenson, Y. Castin, E. Bonderup, and K. Mølmer, Journal of Physics B: Atomic, Molecular and Optical Physics 25, 4195 (1992).

[10] V. S. Voŭtsekhovich, M. V. Danileĭko, A. N. Negriǔko, V. I. Romanenko, and L. P. Yatsenko, Sov. Phys. JETP 72, 219 (1991).

[11] J. Dalibard, Y. Castin, and K. Mølmer, Phys. Rev. Lett. 68, 580 (1992).

[12] K. Mølmer, Y. Castin, and J. Dalibard, JOSA B 10, 524 (1993).

[13] C. Corder, B. Arnold, X. Hua, and H. Metcalf, J. Opt. Soc. Am. B 32, B75 (2015). 
[14] C. Corder, B. Arnold, and H. Metcalf, Phys. Rev. Lett. 114, 043002 (2015).

[15] B. Shore, The Theory of Coherent Atomic Excitation, vol. 1 (Wiley, New York, 1990).

[16] R. Chrétien, Master's thesis, Faculté des Sciences Appliquées, Universitè de Liège, Belgium (2014).

[17] D. A. Steck, Sodium D Line Data (2019), https://steck.us/alkalidata/sodiumnumbers.pdf.

[18] D. A. Steck, Cesium D Line Data (2019), https://steck.us/alkalidata/cesiumnumbers.pdf.

[19] S. M. Freund, M. Römheld, and T. Oka, Phys. Rev. Lett. 35, 1497 (1975).

[20] E. Kyröla and S. Stenholm, Optics Communications 22, 123 (1977). 